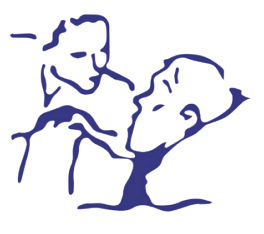

Medicina Paliativa

www.medicinapaliativa.es

ORIGINAL

\title{
Prevalencia de la adherencia terapéutica a opioides mayores en una muestra de pacientes oncológicos avanzados. Estudio piloto
}

\author{
Ramona González-Rubió ${ }^{1}$, Núria Arrarás-Torrelles ${ }^{1}$, Jesús López-Ribes ${ }^{1}$, \\ Eva Barallat-Gimeno ${ }^{* 2}$, Ariadna Gasol-Cudós ${ }^{3,4}$ y Jaume Canals-Sotelo ${ }^{3,5}$
}

\begin{abstract}
${ }^{1}$ Equipo de Soporte Domiciliario, Hospital Santa Maria-Gestió de Serveis Sanitaris, Lleida. ${ }^{2} F a c u l t a d$ de Enfermería y Fisioterapia, Universitat de Lleida. ${ }^{3}$ Institut de Recerca Biomèdica (IRB), Lleida. ${ }^{4}$ Servicio de Oncología Médica, Hospital Universitari Arnau de Vilanova, Lleida. ${ }^{5}$ Equipo de Soporte de Cuidados Paliativos, Hospital Santa Maria-Gestió de Serveis Sanitaris, Lleida. España.
\end{abstract}

Recibido el 14 de febrero de 2017

Aceptado el 24 de junio de 2017

\section{PALABRAS CLAVE \\ Adherencia \\ terapéutica, cuidados paliativos, cáncer, opioides, escalera analgésica de la OMS, test de Morisky-Green- Levine (MMAS-4).}

\begin{abstract}
Resumen
Objetivo: Determinar la prevalencia de adherencia terapéutica a opioides mayores de la escala analgésica de la Organización Mundial de la Salud (OMS) medida con el test 4-ítem Morisky Medication Adherence Scale (MMAS-4), en una muestra de pacientes oncológicos avanzados. Conocer los factores asociados a adherencia terapéutica en este tipo de paciente.

Material y método: Estudio observacional prospectivo realizado en la Región Sanitaria de Lleida entre septiembre de 2013 y marzo de 2014. Se incluyeron 89 pacientes oncológicos avanzados consecutivamente, atendidos por equipos específicos de fin de vida, ya sea en el domicilio (PADES) o bien en la consulta externa de ámbito hospitalario (UFISS). Se utilizó el test autoadministrado MMAS-4.

Resultados: Se incluyeron un total de 89 pacientes de los cuales 48 (54\%) fueron atendidos por PADES y 41 (46\%) por UFISS. Media de edad (70,3 $\pm 14,1$ años). Pacientes varones 64 (72\%). La dosis equivalente de morfina diaria (MEDD) fue de 76,26 $\pm 74,73 \mathrm{mg}$. Un total de 47 pacientes $(52,8 \%)$ presentaron adherencia al tratamiento. Haber recibido analgésicos por vía transdérmica es un factor que contribuye a la mala adherencia terapéutica $(p=0,006)$ y fallar la pregunta $D$ del MMAS-4 junto con género femenino contribuye a la mala adherencia terapéutica $(p=0,027)$.

Discusión: Se detecta un 52,8 \% de adherencia terapéutica a opioides del tercer escalón de la OMS en la muestra estudiada.
\end{abstract}

\footnotetext{
*Autor para correspondencia:

Eva Barallat Gimeno

Universitat de Lleida. Facultat d'Ifermeria; Fisioteràpia. c/ Montserrat Roig, 2. 25198. Lleida, España

Correo electrónico: ebarallat@dif.udl.cat
}

DOI: 10.20986/medpal.2019.1030/2019

1134-248X/ ( 2019 Sociedad Española de Cuidados Paliativos. Publicado por Inspira Network. Todos los derechos reservados. 


\section{KEYWORDS}

Therapeutic

adherence, palliative care, cancer, opioids, WHO analgesic ladder, 4-items Morisky-

Green-Levine tool.

\section{Abstract}

Aim: To determine the prevalence of the therapeutic adherence to strong opioids analgesics (third step of the analgesic ladder of the World Health Organization [WHO]) in a sample of advanced cancer patients using the Morisky-Green-Levine tool (MMAS-4) and to know the factors associated.

Methods: Observational and prospective study carried out in the Sanitary Region of Lleida between September 2013 and March 2014. A total of 89 advanced cancer patients consecutively attended by end-of-life specific teams both at home (PADES) and at the hospital (UFISS) were included. The self-administered 4-items Morisky-Green-Levine test was used.

Results: Mean of age (70, $3 \pm 14,1$ years). Man 64 (72\%). Patients attended by the Home care Team 48 patients (54\%). The Morphine Equivalent Daily Dose (MEDD) was $76.26 \pm 74.73 \mathrm{mg}$. The therapeutic adherence was detected in 47 patients $(52.8 \%)$. Factors related to the route of administration of the analgesic (transdermal, $p=0,006$ ) and between female genre and to fail to question $D$ of the MMAS-4 ( $p=0,027)$ were detected in the multivariate analysis.

Discussion: We detected a prevalence of therapeutic adherence to the analgesics of the third ladder of the WHO of $52,8 \%$ in the sample studied.

González-Rubió R, Arrarás-Torrelles N, López-Ribes J, Barallat-Gimeno E, Gasol-Cudós A, Canals-Sotelo J. Prevalencia de la adherencia terapéutica a opioides mayores en una muestra de pacientes oncológicos avanzados. Estudio piloto. Med Paliat. 2019;26(1):50-54.

\section{Introducción}

Los pacientes oncológicos en situación de enfermedad avanzada deben afrontar multitud de síntomas que pueden afectar distintas esferas (física, emocional, social, espiritual, etc. $)^{1}$. Estos síntomas suelen presentar mayor intensidad a medida que la enfermedad progresa ${ }^{2}$. Por este motivo, es crucial una correcta valoración sintomática llevada a cabo por equipos expertos en cuidados paliativos ${ }^{3}$.

El dolor es uno de los síntomas más temido por enfermos, familiares y equipo asistencial, ya que este síntoma aumenta su prevalencia al avanzar la enfermedad ${ }^{4}$.

En 1986, la Organización Mundial de la Salud (OMS) estableció la piedra de toque para el correcto abordaje del dolor en los enfermos de cáncer mediante la elaboración de la llamada escalera analgésica de la $\mathrm{OMS}^{5}$. En esta, se establece un primer escalón para el dolor de intensidad leve y compuesto por los fármacos no opioides paracetamol y antinflamatorios (AINE); un segundo escalón para el dolor de intensidad moderada con los fármacos opioides menores codeína y tramadol y, finalmente, un tercer escalón para el dolor de intensidad severa y con los fármacos opioides mayores (morfina, fentanilo, metadona, oxicodona y otros) (6,7. $^{\text {. }}$

En 2001 se realizó el "Adherence Meeting" ${ }^{8}$ bajo los auspicios de la OMS, y donde se consensuó una primera definición del concepto adherencia o cumplimiento. Posteriormente, y conjuntamente con los trabajos de Haynes ${ }^{9}$ y Rand ${ }^{10}$, el término adherencia se midió como la medida en la que el comportamiento de una persona (tomar medicación, seguir una dieta y/o ejecutar cambios de estilo de vida) se corresponde con las recomendaciones de su equipo asistencial.

Con todo lo dicho hasta ahora, podemos afirmar que no existe un patrón de oro para medir el fenómeno de la adherencia al tratamiento ${ }^{11,12}$.

Existen distintos métodos de aproximación a la realidad del fenómeno adherencia al tratamiento. Estos métodos de aproximación pueden ser subjetivos u objetivos. El método subjetivo más sencillo es preguntar directamente al paciente ${ }^{13}$, con el riesgo que comporta una sobreestimación de la adherencia. Otro método subjetivo incluye la utilización del cuestionario autoadministrado Four-item Morisky Medication Adherence Scale (MMAS-4), tal como se muestra en la Tabla I. Este valora la adherencia al tratamiento farmacológico. Su valor predictivo evaluado con alfa Cronbach varía entre 0,61 y 0,86 ${ }^{14}$.

Aunque inicialmente las estrategias de aproximación objetivas parecen ser una mejora respecto a los enfoques subjetivos, estas no están exentas de inconvenientes. Un método objetivo es la utilización de las unidades de dosificación, por ejemplo tabletas, que pueden ser utilizadas en cada visita para comprobar la adherencia al tratamiento. Sin embargo, las imprecisiones son comunes y típicamente resultan en una sobrestimación del cumplimiento y en una pérdida importante de información. Una innovación de mejora de esta aproximación objetiva está asociada a las nuevas tecnologías y se denomina monitorización electrónica (Medication Event Monitoring System [MEMS]). Este sistema registra la hora y fecha en la que el contenedor del medicamento se abre y puede describir los distintos patrones en que los pacientes toman sus medicamentos ${ }^{15}$. Desafortunadamente, el coste de estos dispositivos impide su uso generalizado y es de difícil implementación en los servicios de PADES y UFISS.

Tabla I. Cuestionario de Morisky-Green-Levine (4-ítems MMAS)

A. ¿Se olvida alguna vez de tomar la medicación?

B. ¿Toma la medicación a la hora indicada?

C. Cuando se encuentra bien, ¿deja alguna vez de tomar la medicación?

D. Si alguna vez se siente mal, ¿deja de tomar la medicación?

El orden de las respuestas es NO-SÍ-NO-NO. Cualquier error se considera falta de cumplimiento. 
La medición bioquímica es una tercera opción objetiva para evaluar el cumplimiento. La adición de marcadores biológicos no tóxicos puede agregarse a los medicamentos y se puede determinar su presencia en la sangre o en la orina. Los resultados pueden ser equívocos, dado que pueden estar influenciados por una gran variedad de factores individuales (dieta, la absorción y la tasa de excreción) ${ }^{16}$.

La falta de cumplimiento al tratamiento ha sido identificada como una barrera importante para conseguir una buena respuesta analgésica en pacientes oncológicos ${ }^{17}$. Esta barrera es, además, multifactorial e incluye el miedo a ciertos analgésicos ${ }^{18,19}$, factores psicocognitivos ${ }^{19,20}$ (grado de satisfacción conseguido con el tratamiento analgésico), factores relacionados con el propio dolor ${ }^{19,20}$ (intensidad, mejora, significado) y factores sociodemográficos ${ }^{19,20}$.

Existen distintos estudios que muestran la importancia del fenómeno del cumplimiento terapéutico. Así, Miaskowski y cols. ${ }^{17}$ describen niveles de cumplimiento que varían entre el 85 y el $91 \%$ para la medicación basal, hasta el 22-27\% para la medicación de rescate. Asimismo, el grado de cumplimiento puede variar en función del tipo de analgésico utilizado. Enting y cols. ${ }^{21}$ concluyen en su estudio que el cumplimiento terapéutico variaba entre el $59 \%$ para tramadol hasta el 91 \% para los fármacos del tercer escalón de la OMS.

En el trabajo de Valeberg y cols. ${ }^{20}$ se concluye que utilizando test autoadministrados en pacientes oncológicos, solo el $41 \%$ de los pacientes eran adherentes al régimen analgésico prescrito. Utilizando la misma metodología, Tzeng y cols. ${ }^{19}$ concluyen que la mayoría de los pacientes (51\%) mostraban bajos niveles de adherencia a los analgésicos.

Los objetivos de nuestro estudio son los de determinar la prevalencia de adherencia terapéutica a opioides mayores de la escala analgésica de la OMS medida con el test MMAS4 , en una muestra de pacientes oncológicos avanzados, y conocer los factores asociados a adherencia terapéutica en este tipo de paciente.

Adherencia terapéutica y cumplimiento terapéutico son dos términos que pueden utilizarse indistintamente, pues significan lo mismo.

\section{Material y métodos}

Estudio observacional descriptivo de los pacientes atendidos consecutivamente, entre septiembre de 2013 y marzo de 2014, por los equipos de PADES y UFISS.

Este estudio se diseñó como punto de partida para poder calcular el tamaño de la muestra de un posterior estudio basado también en la determinación de la prevalencia del cumplimiento terapéutico en una muestra de pacientes oncológicos avanzados y en tratamiento con fármacos analgésicos del tercer escalón de la OMS.

Criterios de inclusión: pacientes con cáncer avanzado mayores de 18 años que durante la semana previa a su valoración presentaban un dolor basal $\geq 4$ medidos mediante la Escala Visual Analógica de 0-10, que estuvieran recibiendo tratamiento continuado con fármacos analgésicos del tercer escalón de la OMS (morfina, metadona, buprenorfina, oxicodona, fentanilo o hidromorfona) al menos durante la semana previa a la inclusión en el estudio, que no tuvieran deterioro cognitivo valorado con $\leq 3$ errores en el test de Pfeiffer) y que hubieran dado el consentimiento informado.

Variables estudiadas: edad, género, diagnóstico principal, lugar de valoración (PADES o UFISS), polifarmacia (número total de fármacos prescritos), vía de administración de los fármacos analgésicos (oral, transdérmica, subcutánea, transmucosa/sublingual), vivir en núcleo aislado (se consideró núcleo aislado, aquel que no fuera capital de las comarcas de la región sanitaria de Lleida), la dosis equivalente de morfina diaria en mg (MEDD), el índice de Barthel, el test de Pfeiffer, el nivel sociocultural, analfabetismo, la alteración de los sentidos (vista/oído), y los resultados del test MMAS-4.

El análisis estadístico descriptivo de las variables se efectuó con el paquete estadístico SPSS 18 para Microsoft Windows. Se realizó un análisis univariante de los datos obtenidos donde la variable dependiente era la existencia de cumplimiento terapéutico. El análisis se hizo con la prueba t de Student para variables independientes en los parámetros cuantitativos y con la prueba $\chi^{2}$ para parámetros cualitativos. Se efectuó también un análisis multivariante, en concreto una regresión logística, en el que solo se incluyeron las variables que mostraban significación estadística con el modelo univariante. Se estableció la significación estadística en $\mathrm{p}<0,05$.

El trabajo dispone de la autorización del Comité de Ética e Investigación Clínica de referencia (proyecto 1224/2013 aprobado el 25/7/2013).

\section{Resultados}

Se incluyeron un total de 89 pacientes atendidos de manera consecutiva de los cuales 48 (54\%) se atendieron en domicilio. La media de edad de la muestra fue de 70,3 \pm 14,1 [27-93] años. Para los hombres ( $\mathrm{n}=64,72 \%$ ) la media de edad fue de $68,85 \pm 14,9$ [27-92] años mientras que para las mujeres $(n=25,28 \%$ ) la media de edad fue de 74,25 \pm 12,46 [51-93] años, sin significación estadística. En cuanto a la clasificación de los diagnósticos, el más prevalente fue el de origen genitourinario $(n=20,22,5 \%)$ seguido del broncopulmonar $(n=18$, $20,2 \%)$, digestivo superior $(\mathrm{n}=16,18 \%)$, digestivo inferior $(n=14,15,7 \%)$, ORL $(n=9,10,1 \%)$ y otros $(n=12,13,5 \%)$.

La media del test de Barthel fue de 80/100. La media de fármacos prescritos a los pacientes fue de 7,6 para el total de la muestra. La MEDD en mg fue de 76,2 para el total de la muestra ${ }^{22}$.

La vía de administración de los fármacos se distribuyó en función del lugar de atención (Tabla II).

En el análisis del test MMAS-4, 47 pacientes $(52,8 \%)$ cumplen el régimen analgésico mientras que 42 pacientes $(47,2 \%)$ no cumplen.

En el análisis univariante se halló una relación estadísticamente significativa entre la variable no cumplimiento terapéutico y las variables vía transdérmica $(p=0,004)$ y género femenino $(p=0,040)$, así como entre las variables género femenino y errar a la pregunta $D$ en el MMAS-4 ( $p=0,025)$.

En cambio, en el análisis multivariante existe una relación estadísticamente significativa entre las variables no cumplimiento terapéutico y recibir los fármacos opioides por vía transdérmica $(p=0,006)$ y entre las variables género femenino y errar en la pregunta $D$ en el MMAS-4 ( $p=0,027)$.

En la Tabla III podemos ver la distribución de los errores a 
Tabla II. Vías de administración de los distintos fármacos en función del equipo

\begin{tabular}{ccccc}
\hline Vía de administración & Vía oral & Vía subcutánea & Vía transmucosa & Vía transdérmica \\
\hline UFISS $(\mathrm{n}=41)$ & $36(87,8 \%)$ & $0(0 \%)$ & $7(17 \%)$ & $5(12,2 \%)$ \\
PADES $(\mathrm{n}=48)$ & $38(79,1 \%)$ & $6(12,5 \%)$ & $9(18,7 \%)$ & $12(25 \%)$ \\
Total $(\mathrm{n}=89)$ & $74(83,1 \%)$ & $6(6,7 \%)$ & $16(18 \%)$ & $17(19,1 \%)$ \\
\hline
\end{tabular}

Tabla III. Errores en el MMAS-4 en función del equipo

\begin{tabular}{ccccc}
\hline Errores MMAS-4 & Pregunta A & Pregunta B & Pregunta C & Pregunta D \\
\hline UFISS & 9 & 13 & 10 & 7 \\
PADES & 10 & 9 & 10 & 17 \\
Total & 19 & 22 & 20 & 24 \\
\hline
\end{tabular}

las distintas preguntas del MMAS-4 en función del lugar de atención de los pacientes.

\section{Discusión}

La no adherencia terapéutica se define como el hecho en que el comportamiento de un paciente no coincide con el consejo de los profesionales sanitarios. Solucionar el problema de la no adherencia al tratamiento prescrito podría constituir una mejora sanitaria equiparable a la magnitud de cualquier avance biomédico ${ }^{23}$.

La eficacia de los fármacos para el buen control de la enfermedad depende precisamente de la adherencia por parte del paciente al régimen terapéutico prescrito. A pesar de esta afirmación, diversos estudios ${ }^{20}$ muestran que hasta un $50 \%$ de los pacientes abandonan el tratamiento a los seis meses de haberlo iniciado.

Aunque el trabajo original de los autores del MMAS-4 se basó en el cumplimiento terapéutico en pacientes hipertensos, posteriormente se ha trasladado a otros campos de atención sanitaria donde se ha evidenciado, una vez más y en línea con la OMS, la importancia de monitorizar el cumplimiento terapéutico como la piedra angular sobre la que se basará la efectividad de dicho tratamiento.

Existen pocos trabajos centrados exclusivamente en el estudio del cumplimiento terapéutico relacionado con fármacos analgésicos del tercer escalón de la escalera analgésica de la OMS y, según la revisión llevada a cabo por los autores, ninguno en nuestro ámbito geográfico.

El resultado obtenido con un cumplimiento terapéutico del $52,5 \%$ de los pacientes no deja de sorprendernos cuando nos centramos en el tratamiento del síntoma dolor. El dolor, una vez correctamente evaluado, tiene un tratamiento a priori efectivo basado en la administración de fármacos analgésicos en función de su intensidad. No obstante, este resultado es similar al de Valeberg y cols. ${ }^{20}$, que obtuvieron un $46 \%$ de cumplimiento a partir de una muestra de 174 pacientes oncológicos. En este estudio se utilizó también el MMAS-4 como instrumento de valoración del cumplimiento terapéutico.

Nuestro resultado concuerda también con el trabajo de
Tzeng y cols. ${ }^{19}$, quienes utilizando la versión Taiwanesa del MMAS- 4 obtuvieron un nivel de adherencia del $49 \%$, mucho más cercana al 52,5\% que hemos encontrado en nuestro estudio.

Miaskowski y cols. ${ }^{17}$, con una muestra de 65 pacientes, diferencian en su trabajo entre los fármacos opioides utilizados como tratamiento basal y los utilizados como rescate, hecho que los autores no hemos contemplado. En este caso, los niveles de adherencia son muy superiores a los que hemos obtenido en nuestro trabajo $(85,5-$ $90,8 \%$ vs. $52,5 \%$ ). Cabe destacar que Miaskowski y cols. únicamente incluyeron pacientes oncológicos con dolor relacionado con la existencia de metástasis óseas y que el punto de corte de la intensidad del dolor fue de 2,5, mientras que en nuestro trabajo el punto de corte fue de 4 (EVA $\geq 4)$.

Los tamaños de la muestra de los estudios consultados son similares al que aquí presentamos.

\section{Limitaciones del estudio}

En primer lugar, una limitación de nuestro estudio puede ser el hecho de no diferenciar entre la medicación pautada fija y la medicación pautada como rescate, pues el comportamiento puede mostrarse diferente cuando interviene la variable de la voluntad del paciente en decidir si debe o no tomar un medicamento.

En segundo lugar, podría ser interesante profundizar en el concepto intencional y no intencional, pues el concepto de adherencia no ha de ser visto como un constructo monolítico y sí como un paso más hacia el reconocimiento como un proceso consciente o inconsciente de diferentes dimensiones de la adherencia ${ }^{17,24}$.

Finalmente, el uso del MMAS-4 como instrumento de detección de la adherencia al tratamiento farmacológico, si bien es sencillo y barato, tiende a sobreestimar la adherencia de los pacientes estudiados.

La fortaleza de nuestro trabajo está basada en dos puntos: - El primero es que es un punto de partida para la realización de otros trabajos, tanto cuantitativos como cua- 
litativos, para conocer mejor los mecanismos que subyacen al fenómeno del cumplimiento/no cumplimiento terapéutico.

- El segundo es, precisamente, el resultado obtenido. Aun con las limitaciones antes descritas, el fenómeno del cumplimiento terapéutico debería ser valorado en la práctica clínica diaria con la finalidad de mejorar el abordaje del síntoma dolor en los pacientes oncológicos avanzados.

\section{Responsabilidades éticas}

Los autores declaran que para esta investigación no se han realizado experimentos en seres humanos ni en animales.

Los autores declaran que han seguido los protocolos de su centro de trabajo sobre la publicación de datos de pacientes.

Los autores declaran que en este artículo no aparecen datos de pacientes.

Los autores declaran que no existe conflicto de intereses.

\section{Bibliografía}

1. Syrala KL, Jensen MP, Mendoza ME, Yi JC, Fisher HM, keefe FJ. Psychological and behavioral approaches to cancer pain management. J Clin Oncol. 2014;32(16):1703-11.

2. Black B, Herr K, Fine P, Sanders S, Tang X, Bergen-Jackson K, et al. The relationships among pain, non pain symptoms, and quality of life measures in older adults with cancer receiving hospice care. Pain Med. 2011;12(6):880-9.

3. Shin J, Temel J. Integrating palliative care: when and how? Curr Opin Pulm Med. 2013;19(4):344-9.

4. Meldrum $M$. The ladder and the clock: cancer pain and public policy at the end of the twentieth century. J Pain Symptom Manage. 2005;29(1):41-54.

5. World Health Organization. Cancer pain relief. Geneva: World Health Organization; 1986.

6. Zech DF, Grond S, Lynch J, Hertel D, Lehmann KA. Validation of World Health Organization Guidelines for cancer pain relief: A 10-year prospective study. Pain. 1995;63(1):65-76.

7. Vargas-Schaeffer $\mathrm{G}$. Is the WHO analgesic ladder still valid? Twenty-four years of experience. Can Fam Physician. 2010;56(6):514-7.

8. Sabate E. WHO Adherence Meeting Report. Geneva, World Health Organization; 2001.

9. Haynes RB. Determinants of compliance: The disease and the mechanics of treatment. Baltimore MD: Johns Hopkins University Press; 1979.
10. Rand CS. Measuring adherence with therapy for chronic diseases: implications for the treatment of heterozygous familial hypercholesterolemia. Am J Cardiol. 1993 Sep 30;72(10): 68D-74D.

11. Timmreck TC, Randolph JF. Smoking cessation: clinical steps to improve compliance. Geriatrics 1993;48(4):63-6.

12. Farmer KC. Methods for measuring and monitoring medication regimen adherence in clinical trials and clinical practice. Clin Ther. 1999;21(6):1074-90.

13. Norell SE. Accuracy of patient interviews and estimates by clinical staff in determining medication compliance. Social Science \& Medicine - Part E, Medical Psychology 1981;15(1):57-61.

14. Erickson SR, Coombs JH, Kirking DM, Azimi AR. Compliance from self-reported versus pharmacy claims data with metered-dose inhalers. Ann Pharmacother 2001;35(9):997-1003.

15. Oldenmenger WH, Echteld MA, de Wit R, Sillevis Smitt PA, Stronks DL, Stoter G, et al. Analgesic adherence measurement in cancer patients: Comparison between electronic monitoring and diary. J Pain Symptom Manage. 2007;34(6):639-47.

16. Bosworth, HB. Medication treatment adherence. In: Bosworth HB, Oddone EZ, Weinberger M, editors. Patient treatment adherence: Concepts, intervention, and measurement. Mahwah, NJ: Lawrence Erlbaum Associates; 2006. p. 147-94.

17. Miaskowski C, Dodd MJ, West C, Paul SM, Tripathy D, Koo P, et al. Lack of adherence with the analgesic regimen: A significant barrier to effective cancer pain management. J Clin Oncol. 2001;19(23):4275-9.

18. Gunnarsdottir S, Donovan HS, Serlin RC, Voge C, Ward S. Patient-related barriers to pain management: The Barriers Questionnaire II (BQ-II). Pain. 2002;99(3):385-96.

19. Tzeng JI, Chang CC, Chang HJ, Lin CC. Assessing analgesic regimen adherence with the Morisky Medication Adherence Measure for Taiwanese patients with cancer pain. J Pain Symptom Manage. 2008;36(2):157-66.

20. Valeberg BT, Miaskowski C, Hanestad BR, Bjordal K, Moum T, Rustoen T. Prevalence rates for and predictors of self-reported adherence of oncology outpatients with analgesic medications. Clin J Pain. 2008;24(7):627-36.

21. Enting RH, Oldenmenger WH, Van Gool AR, van der Rijt CC, Sillevis Smitt PA. The effects of analgesic prescription and patient adherence on pain in a Dutch outpatient cancer population. J Pain Symptom Manage. 2007;34(5):523-31.

22. Chatham MS, Dodds Ashley ES, Svengsouk JS, Juba KM. Dose ratios between high dose oral morphine or equivalents and oral methadone. J Palliat Med. 2013;16(8):947-50.

23. Sabate E. Adherence to long-term therapies: evidence for action. Geneva, Switzerland: World Health Organization; 2003.

24. Lowry KP, Dudley TK, Oddone EZ, Bosworth HB. Intentional and unintentional non-adherence to antihypertensive medication. Ann Pharmacother. 2005;39(7-8):1198-203. 